\title{
Multiscale Characterization Reveals Oligomerization Dependent Phase Separation of Primer-independent RNA Polymerase nsp8 From SARS-CoV-2
}

Jinxin Xu

Guangzhou Institutes of Biomedicine and Health, Chinese Academy of Sciences

\section{Xin Jiang}

Neutron Science Platform, Songshan Lake Materials Laboratory

\section{Yan Dong}

Guangzhou Institutes of Biomedicine and Health

\section{Changli Ma}

Spallation Neutron Source Science Center, Dongguan

\section{Hanqiu Jiang}

Spallation Neutron Source Science Center, Dongguan

\section{Taisen Zuo}

Spallation Neutron Source Science Center, Dongguan

\section{Yulong Zhang}

Guangzhou Institutes of Biomedicine and Health

\section{Rui Chen}

University of Science and Technology of China

\section{Yubin Ke}

Spallation Neutron Source Science Center, Dongguan

He Cheng

Spallation Neutron Source Science Center, Dongguan

\section{Howard Wang}

Neutron Science Platform, Songshan Lake Materials Laboratory,

Jinsong Liu ( $\square$ liu_jinsong@gibh.ac.cn )

Guangzhou Institutes of Biomedicine and Health https://orcid.org/0000-0003-2317-0558

\section{Article}

Keywords: RNA replication, oligomerization, separation of primer-independent RNA, polymerase nsp8, SARS-CoV-2

Posted Date: December 15th, 2021 
DOI: https://doi.org/10.21203/rs.3.rs-1144688/v1

License: (c) (1) This work is licensed under a Creative Commons Attribution 4.0 International License. Read Full License

Version of Record: A version of this preprint was published at Communications Biology on September 7th, 2022. See the published version at https://doi.org/10.1038/s42003-022-03892-x. 


\section{Abstract}

RNA replication and transcription machinery is an important drug target for fighting against coronavirus. Non-structure protein nsp8 was proposed harboring primase activity. However, the RNA primer synthesis mechanism of nsp8 is still largely unknown. Here, we purified dimer and tetramer forms of SARS-CoV-2 nsp8. Combined with DLS, SANS and thermo-stability analysis, we found that both dimer and tetramer become loosened and destabilized with decreasing salt concentration, and the dimer form is more stable than the tetramer form. Further investigation showed that nsp8 dimer and tetramer can undergo phase separation but exhibit different phase separation behaviors. nsp8 dimer can form liquid-like droplets in the buffer with a low concentration of $\mathrm{NaCl}$; phase separation of nsp8 tetramer depends on the assistance of RNA. Our findings on different phase separation behaviors of nsp8 dimer and tetramer could provide novel insight into the primer synthesis mechanism in coronavirus and facilitate developing novel therapeutic agents against SARS-CoV-2.

\section{Introduction}

Infection of coronaviruses, a group of positive-strand RNA viruses, is a great threat to the human health. The current pandemic coronavirus disease 2019 (COVID-19) is caused by a new coronavirus SARS-CoV-2 ${ }^{1}$. Despite extensive applications of vaccine, COVID-19 remains a threat to the global economy and health, due to the shortage of effective therapeutic agents against SARS-CoV-2. Considering the essential role in the life cycle of RNA viruses and the lack of homolog in host cells, RNA replication and transcription machinery is an attractive therapeutic target against SARS-CoV- ${ }^{2}, 3$. At least two RNAdependent RNA polymerase (RdRp) are encoded by the coronavirus genome, including primer-dependent RdRp nsp $12{ }^{4}$ and primer-independent RdRp nsp8 ${ }^{5,6}$. Recent structural studies on SARS-CoV nsp $12^{7}$ and SARS-CoV-2 nsp $12^{8,9}$ have characterized the RNA elongation mechanism of nsp12. It was proposed that nsp8 is capable of de novo RNA synthesis with an ssRNA template, and provides primers required for nsp $12^{5}$. Although structures of nsp8 from several coronaviruses were reported, the mechanism of nsp8 de novo RNA synthesis is still largely unknown.

Concentrating reactive molecules in condensates via liquid-liquid phase separation (LLPS) was considered a powerful mechanism for accelerating biochemical reactions. It was well established that weak interactions mediated by intrinsically disordered regions (IDR) of proteins are critical for phase separation ${ }^{10}$. Sequence analysis showed that SARS-CoV-2 nsp8 contains potential IDR at the $\mathrm{N}$ terminus (Fig. S1), which is critical for RNA binding ${ }^{11}$, and contains conserved catalytic D/ExD/E motif as characterized in SARS-CoV nsp8 ${ }^{6}$. Structural analysis also showed that free $\mathrm{N}$ terminus is flexible in several reported structures ${ }^{12}, 13$, implying that nsp8 may undergo phase separation. Here, we aim to elucidate the property and phase separation behavior of SARS-CoV2-nsp8 in solution and live cells, providing novel insights on primer synthesis mechanism in coronavirus.

\section{Results And Discussion}




\section{SARS-CoV-2 nsp8 forms dimer and tetramer in solution}

We expressed and purified SARS-CoV-2 nsp8 with a C terminal 8xHis-tag. During the purification process, we found that nsp8 forms multi-oligomer and nucleic acid contamination when the purification buffer contains $300 \mathrm{mM} \mathrm{NaCl}$. To avoiding the contamination by nucleic acids, the concentration of $\mathrm{NaCl}$ in purification buffer was increased to $1 \mathrm{M}$. It is interesting that nsp8 was eluted from gel filtration column as two peaks (Fig. 1A). The oligomerization state of nsp8 from two elution peaks was analyzed using dynamic light scattering (DLS) and small angle neutron scattering (SANS). The calculated molecular weight of nsp8 from the first and second elution peak was 90-93 kDa and 40-49 kDa, respectively (Fig. 1B). As the theoretical molecular weight of nsp8-His is $22.98 \mathrm{kDa}$, data indicate that in addition to the purified dimer of nsp8 as reported previously ${ }^{14}$, a tetramer form of nsp8 was identified. Then, we characterized the shape of nsp8 dimer and tetramer based on the DLS and SANS measurements. Hydrodynamic radius $\left(R_{\mathrm{h}}\right)$ as measured by DLS reflects the solvated protein size, while radius of gyration $\left(R_{\mathrm{g}}\right)$ as measured by SANS reflects mostly the compositional distribution of hydrogenated protein molecules. The ratio of $R_{\mathrm{g}}$ to $R_{\mathrm{h}}\left(R_{\mathrm{g}} / R_{\mathrm{h}}\right)$ offer the shape information of protein molecules ${ }^{15}$. The larger $R_{\mathrm{g}} / R_{\mathrm{h}}$ values of nsp8 dimers than those of nsp8 tetramers imply the variation of the shape of molecules from non-spherical to globular (Fig. 1C and D).

\section{Destabilization of both nsp8 dimer and tetramer as salt concentration decreasing}

Interestingly, DLS analysis demonstrated that the $R_{\mathrm{h}}$ of nsp8 dimer and tetramer increased with decreasing $\mathrm{NaCl}$ concentration (Fig. 1C). This corroborated well with the SANS measurements that revealed the increase of the $R_{\mathrm{g}}$ at lower $\mathrm{NaCl}$ concentrations for both nsp8 dimer and tetramer (Fig. 1D). Both $R_{\mathrm{h}}$ and $R_{\mathrm{g}}$ of dimers and tetramers increase noticeably when the concentration of $\mathrm{NaCl}$ is decreased, indicating a looser structure in the buffer containing low concentration of $\mathrm{NaCl}$. These data may suggest that lowering salt concentration destabilizes the structure of both nsp8 dimers and tetramers. To test the hypothesis, we assessed the thermo-stability of nsp8 dimer and tetramer, and revealed that both the melting temperature $\left(T_{\mathrm{m}}\right)$ and the onset temperature of aggregation $\left(T_{\mathrm{agg}}\right)$ indeed decreased at lower $\mathrm{NaCl}$ concentration for both nsp8 dimer and tetramer (Fig. 1E and F). Furthermore, quantitative comparison of $T_{\mathrm{m}}$ and $T_{\text {agg }}$ values of the two forms of nsp8 indicates that the dimer form is more stable than the tetramer one.

\section{Different phase separation behavior of nsp8 dimer and tetramer}

The expanded size and decreased stability with decreasing $\mathrm{NaCl}$ concentration may imply that nsp8 dimer and tetramer can undergo phase separation at low $\mathrm{NaCl}$ concentration. To test the hypothesis, we directly diluted nsp8 with a low $\mathrm{NaCl}$ concentration buffer. Liquid-like droplets formed in $1 \mathrm{mg} / \mathrm{mL} \mathrm{nsp} 8$ solution containing $50 \mathrm{mM} \mathrm{NaCl}$ (Fig. 2A). When the concentration of $\mathrm{NaCl}$ was increased to $100 \mathrm{mM}$, nsp8 dimer failed to undergo phase separation at $1 \mathrm{mg} / \mathrm{mL}$ (Fig. 2A), whereas at $2 \mathrm{mg} / \mathrm{mL}$, nsp8 dimer also formed liquid-like droplet in the buffer containing $100 \mathrm{mM} \mathrm{NaCl}$ (Fig. 2B). For fluorescent microscopy observation, nsp8 was labeled with His-tag labeling dye RED-tris-NTA. Fluorescent microscopy confirmed 
that nsp8 dimers form liquid-like droplets at $1 \mathrm{mg} / \mathrm{mL} \mathrm{nsp} 8$ in the $50 \mathrm{mM} \mathrm{NaCl}$ buffer, and $\mathrm{nsp} 8$ condensates can be dissolved upon increasing the $\mathrm{NaCl}$ concentration to $275 \mathrm{mM}$ by mixing nsp8 dimer $(1 \mathrm{mg} / \mathrm{mL}$ ) dissolved in $500 \mathrm{mM} \mathrm{NaCl}$ with volume ratio 1: 1 (Fig. 2C). The liquid-like nature of the droplet was further confirmed by fluorescence recovery after photobleaching (FRAP) experiment as rapid recovery of fluorescence was observed after photobleaching (Fig. 2D). These indicated that nsp8 dimer undergoes liquid-liquid phase separation in solution in a protein and $\mathrm{NaCl}$ concentration dependent manner.

The thermal stability analysis clearly showed that the nsp8 tetramer is significantly less stable than the dimer (Fig. 1E and F). Then, we tested whether nsp8 tetramers exhibit different phase separation behaviors compared to the dimer. Consistent with our hypothesis, nsp8 tetramer forms solid-like sediments instead of liquid-like droplet at concentration of $1 \mathrm{mg} / \mathrm{mL}$ in the buffer containing $50 \mathrm{mM}$ or $100 \mathrm{mM} \mathrm{NaCl}$ (Fig. S2). Solid-like condensates can still be observed even as the concentration of nsp8 tetramer decreased to $0.25 \mathrm{mg} / \mathrm{mL}$ with low $\mathrm{NaCl}$ concentration, which can be reversed by simply increasing $\mathrm{NaCl}$ concentration to $275 \mathrm{mM}$ (Fig. 2E).

To investigate whether the $\mathrm{N}$ terminal IDR is essential for phase transformation, we carried out a truncation of $n s p 8$ by deleting the $\mathrm{N}$ terminal 76 residues (nsp8 $\Delta$ N76). Similar to the wild type nsp8, nsp8 $\Delta$ N76 also forms homodimer and tetramer in solution (Fig. S3). However, the deletion of $\mathrm{N}$ terminal 76 residues unequivocally abolished droplet formation of nsp8 dimer (Fig. 2F), suggesting the importance of the $\mathrm{N}$ terminal IDR for the LLPS of the nsp8 dimer. Interestingly, with the deletion of $\mathrm{N}$ terminal 76 residues, nsp8 tetramer mutants were less aggregated at $50 \mathrm{mM} \mathrm{NaCl}$ compared to the wild type nsp8 tetramer (Fig. 2G). The observation suggested that $\mathrm{N}$ terminal IDR play a key role in overaggregation of nsp8 tetramers at decreasing $\mathrm{NaCl}$ concentration.

\section{RNA modulates LLPS of nsp8 tetramer}

$\mathrm{N}$-terminus of nsp8 is a defined RNA binding motif, which can be stabilized by binding with RNA 8,12 . We speculated that RNA binding may induce nsp8 tetramer transition from solid-like condensate to liquidliquid phase separation at low $\mathrm{NaCl}$ concentration. To confirm this, we designed a 12-nt ssRNA (R12) with sequence derived from SARS-CoV-2 genome adjacent to poly (A). When mixing RED-tris-NTA labeled nsp8 tetramer $(0.25 \mathrm{mg} / \mathrm{ml})$ with R12, liquid-like droplet can be observed with RNA concentration up to $75 \mu \mathrm{M}$ (Fig. 3A). To verify whether RNA modulating LLPS of nsp8 tetramer depends on the sequence and the length, LLPS experiments were performed in the presence of 12-nt poly $\mathrm{U}(\mathrm{U} 12)$ or 22-nt poly $\mathrm{U}$ (U22) (Fig. 3A). We found that, when mixed with $75 \mu \mathrm{M} \mathrm{U12,} \mathrm{nsp8} \mathrm{tetramer} \mathrm{still} \mathrm{forms} \mathrm{solid-like} \mathrm{structure}$ instead of liquid-like droplet. However, nsp8 tetramer turned to liquid-like droplet when mixing with 22-nt poly U (U22) at concentration as low as $7.5 \mu \mathrm{M}$. Thus, data suggested that LLPS of nsp8 tetramer depend on both RNA sequence and length. To confirm that the phase separated droplets were formed by nsp8 binding with RNA, we synthesized 6-FAM labeled R12. Fluorescent microscopy demonstrated that nsp8 and RNA were co-localized in the phase separated droplet (Fig. 3B). 


\section{nsp8 forms condensates in living cells}

Then, we asked whether nsp8 also undergoes liquid-liquid phase separation in live human cell line. To address this notion, nsp8 fusion with monomeric red fluorescent protein mCherry at C-terminal (nsp8mCherry) was over-expressed in Hela cells. By imaging this cell line using fluorescent microscopy, we observed that over-expressed nsp8 could form condensates in cytoplasm, while over-expressed mCherry was diffuse in the entire cell (Fig. 4). Taken together, results suggested that SARS-CoV-2 nsp8 undergo phase separation in solution, as well as in live cells.

\section{Conclusions}

In summary, we have identified the dimer and tetramer forms of nsp8, which undergo phase transformation at the low concentration of $\mathrm{NaCl}$ in aqueous solutions. However, they exhibit distinct phase separation behaviors: nsp8 dimer form separated liquid phases depending only on concentrations of the protein and salt, whereas nsp8 tetramers form solid-like aggregates at the low salt concentration, while transforming to liquid-like droplets with the addition of RNA. Furthermore, nsp8-tetramer-induced LLPS depend on both the sequence and length of RNAs. This study implies the significance of quantifying the degree of disorder in proteins and the nature of interaction among protein and RNA, which can facilitate the development of mechanistic models on nsp8 de novo RNA synthesis and potential therapeutic strategies against the SARS-CoV-2.

\section{Methods}

\section{Protein expression and purification}

SARS-CoV-2 nsp8 and its truncated mutant were cloned into pET21a with C-terminal $8 \mathrm{x}$ His tag. Proteins were expressed in E. coli strain BL21 (DE3) at $16{ }^{\circ} \mathrm{C}$ overnight and induced with $0.1 \mathrm{mM}$ IPTG. Bacteria were collected and re-suspended in lysis buffer (20 mM HEPES pH7.4, $1 \mathrm{M} \mathrm{NaCl}, 10 \mathrm{mM}$ imidazole, $7 \mathrm{mM}$ imidazole). Cells were lysed by high pressure homogenization. After centrifugation, supernatant was loaded onto Ni-NTA resin. After washed with $70 \mathrm{mM}$ imidazole, the bounded protein was eluted with 300 $\mathrm{mM}$ imidazole. Proteins were further purified by gel filtration using a HiLoad superdex 200 (for wild type nsp8) or 75 (for truncated nsp8) equilibrated against buffer of $20 \mathrm{mM} \mathrm{HEPES} \mathrm{pH} \mathrm{7.4,} \mathrm{0.5} \mathrm{M} \mathrm{NaCl.}$

\section{Small-angle neutron scattering (SANS)}

Small angle neutron scattering measurements were performed at the China spallation neutron source (CSNS). $10 \mathrm{mg} / \mathrm{mL}$ nsp8 samples in D2O buffer were measured in quartz cells with $2 \mathrm{~mm}$ optical path length. The scattering experiment times of buffers and samples are $90 \mathrm{~min}$. And the empty beam and empty quartz cell were measured $15 \mathrm{~min}$ for data reduction. Transmissions were measured for $10 \mathrm{~min}$ for each sample and the empty beam. All measurements were carried out at $289 \mathrm{~K}$.

The presented SANS data were reduced and corrected for sample transmission, cell scattering and detector background using reduction algorithms developed based on Mantid framework ${ }^{16}$ provided by 
the instrument. The neutron scattering intensity has been calibrated to absolute intensity using a secondary standard, Bates poly. The SasView (https://www.sasview.org/) software was employed to calculate the radius of gyration $\mathrm{Rg}$ of all samples by Guinier approximation.

\section{Dynamic light scattering (DLS)}

The DLS analyses were performed using DynaPro NanoStarTM (Wyatt Technologies). The quartz cells filled with $100 \mu \mathrm{L} 1 \mathrm{mg} / \mathrm{mL}$ nsp8 sample solution for DLS measurement. The samples were measured though $100 \mathrm{~mW}$ He-Ne laser, $\lambda 0=660 \mathrm{~nm}, \theta=90^{\circ} .10$ times successive measurements were performed per sample for average to get the DLS data. The DYNAMICS software (Wyatt Technologies) was employed to analyze the DLS data to get the RH of all samples.

\section{Thermo-stability analysis}

SARS-CoV-2 nsp8 dimer and tetramer with concentration $1 \mathrm{mg} / \mathrm{ml}$ were dissolved in $20 \mathrm{mM}$ HEPES pH 7.4 , and $150 \mathrm{mM} / 250 \mathrm{mM} / 500 \mathrm{mM} \mathrm{NaCl}$. Thermo-stability analysis was performed with Prometheus NT.48 instrument (Nano Temper Technologies). The Scan temperature was increased linearly with a rate of $1^{\circ} \mathrm{C}$ per min. Melting temperature $(\mathrm{Tm})$ and onset temperature of aggregation (Tagg) were analyzed with PR.ThermControl software.

\section{Imaging of SARS-CoV-2 nsp8 or RNA complex in solution}

For Differential interference contrast (DIC) imaging of SARS-CoV-2 nsp8, the proteins were spotted onto a glass slide. Imaging was performed with Leica DM4 B.

For fluorescence imaging of SARS-CoV-2 nsp8, the proteins were labeled with His-tag labeling kit by mixing protein and RED-tris-NTA 2nd Generation dye with mole ratio about 600:1, and incubating at room temperature for $15 \mathrm{~min}$. To investigate the effect of RNA on phase separation of SARS-CoV-2 nsp8 tetramer, RNA was added to the RED-tris-NTA labeled protein. ssRNA sequences used in this study, R12: rGrArGrArArUrGrArCrArArA, U12: rUrUrUrUrUrUrUrUrUrUrUrU, U22:

rUrUrUrUrUrUrUrUrUrUrUrUrUrUrUrUrUrUrUrUrUrU. For co-localization experiments, R12 was labeled with 6FAM at 5 ' terminal. Before imaging, $10 \mu \mathrm{l}$ aliquot of sample was placed into a well of glass bottom 384 well plates. Imaging was performed with Zeiss LSM 800 Confocal Laser Scanning Microcopy.

\section{Live imaging}

Hela cells were grown on glass bottom culture dish, and cultured in DMEM medium supplemented with $10 \%$ FBS. Cells were transfected with Lipofectamine 2000 following manufacturer's instructions. Before imaging, culture medium was replaced with phenol red-free DMEM supplemented with $10 \%$ FBS. Imaging was performed with Laser Scanning Confocal Microscopy Leica SP8 X STED.

\section{Declarations}




\section{Acknowledgment}

The work was financially supported by Youth Innovation Promotion Association of the Chinese Academy of Sciences (2018390) to J. Xu., Guangzhou Institute of Respiratory Health Open Project (Funds provided by China Evergrande Group) (2020GIRHHMS08), Project of The State Key Laboratory of Respiratory Disease (SKLRD-Z-202009, SKLRD-Z-202213), and Guangdong Provincial Key Laboratory of Biocomputing (2016B030301007). We gratefully acknowledge the China Spallation Neutron Source (CSNS) for the SANS measurements and the Songshan Lake Deuteration Facility (SLDF) for materials and supplies. We would like to thank the SANS beamline stuffs for their support in SANS measurements and the data reduction. We thank Dr. H. Eric Xu for providing the expressing plasmid of the SARS-CoV-2 nsp8.

\section{Contributions}

J.L, H.W, J.X and X.J designed experiment. J.X, X.J, Y.D, C.M, H.J, T.Z, Y.Z, R.C, Y.K and H.C performed experiment. J.L, H.W, J.X and X.J wrote and edited the manuscript.

\section{Conflict of interest}

The authors declare no competing interests.

\section{References}

1. Wu, F. et al. A new coronavirus associated with human respiratory disease in China. Nature 579, 265269 (2020).

2. Zhu, W. et al. RNA-Dependent RNA Polymerase as a Target for COVID-19 Drug Discovery. Slas Discov 25, 1141-1151 (2020).

3. Jiang, Y., Yin, W. \& Xu, H.E. RNA-dependent RNA polymerase: Structure, mechanism, and drug discovery for COVID-19. Biochemical and biophysical research communications 538, 47-53 (2021).

4. Velthuis, A.J.W.T., Arnold, J.J., Cameron, C.E., van den Worm, S.H.E. \& Snijder, E.J. The RNA polymerase activity of SARS-coronavirus nsp12 is primer dependent. Nucleic Acids Res $\mathbf{3 8}, \mathbf{2 0 3 - 2 1 4}$ (2010).

5. Imbert, I. et al. A second, non-canonical RNA-dependent RNA polymerase in SARS coronavirus. The EMBO journal 25, 4933-4942 (2006).

6. Velthuis, A.J.W.T., van den Worm, S.H.E. \& Snijder, E.J. The SARS-coronavirus nsp7+nsp8 complex is a unique multimeric RNA polymerase capable of both de novo initiation and primer extension. Nucleic Acids Res 40, 1737-1747 (2012).

7. Kirchdoerfer, R.N. \& Ward, A.B. Structure of the SARS-CoV nsp12 polymerase bound to nsp7 and nsp8 co-factors. Nature communications 10, 2342 (2019).

8. Wang, Q. et al. Structural Basis for RNA Replication by the SARS-CoV-2 Polymerase. Cell 182, 417428 e413 (2020). 
9. Hillen, H.S. et al. Structure of replicating SARS-CoV-2 polymerase. Nature 584, 154-156 (2020).

10. Alberti, S., Gladfelter, A. \& Mittag, T. Considerations and Challenges in Studying Liquid-Liquid Phase Separation and Biomolecular Condensates. Cell 176, 419-434 (2019).

11. Xiao, Y.B. et al. Nonstructural Proteins 7 and 8 of Feline Coronavirus Form a 2:1 Heterotrimer That Exhibits Primer-Independent RNA Polymerase Activity. J Viro/ 86, 4444-4454 (2012).

12. Zhang, C., Li, L., He, J., Chen, C. \& Su, D. Nonstructural protein 7 and 8 complexes of SARS-CoV-2. Protein science : a publication of the Protein Society 30, 873-881 (2021).

13. Biswal, M. et al. Two conserved oligomer interfaces of NSP7 and NSP8 underpin the dynamic assembly of SARS-CoV-2 RdRP. Nucleic Acids Res 49, 5956-5966 (2021).

14. Wilamowski, M. et al. Transient and stabilized complexes of Nsp7, Nsp8, and Nsp12 in SARS-CoV-2 replication. Biophysical journal 120, 3152-3165 (2021).

15. Kok, C.M. \& Rudin, A. Relationship between the Hydrodynamic Radius and the Radius of Gyration of a Polymer in Solution. Makromol Chem-Rapid 2, 655-659 (1981).

16. Du, R. et al. Data reduction for time-of-flight small-angle neutron scattering with virtual neutrons. Instrum Sci Technol 45, 541-557 (2017).

\section{Figures}



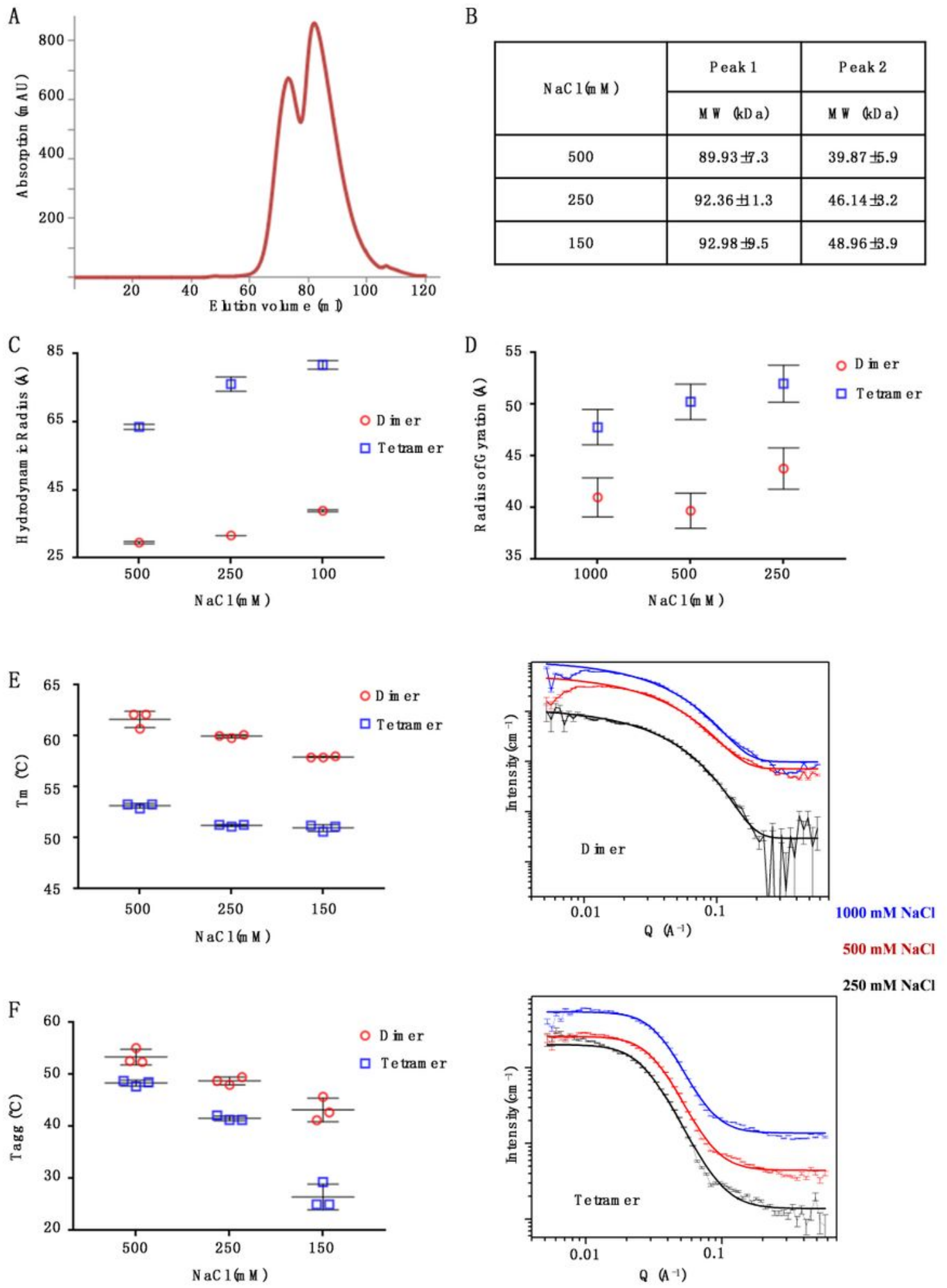

\section{Figure 1}

Characterization of SARS-CoV-2 nsp8. (A) Gel filtration analysis of nsp8. (B) Molecular weight determination of nsp8 from two eluted peaks using DLS. (C). Hydrodynamic radius of nsp8 dimers and tetramers. (D) Radius of gyration (Rg) of nsp8 dimers and tetramers in buffers containing different concentrations of $\mathrm{NaCl}$. The Rg quantities were obtained from model fitting to the SANS spectra of 
corresponding solutions as shown in lower panels. (E) Melting and (F) aggregation temperature of nsp8 dimers and tetramers.

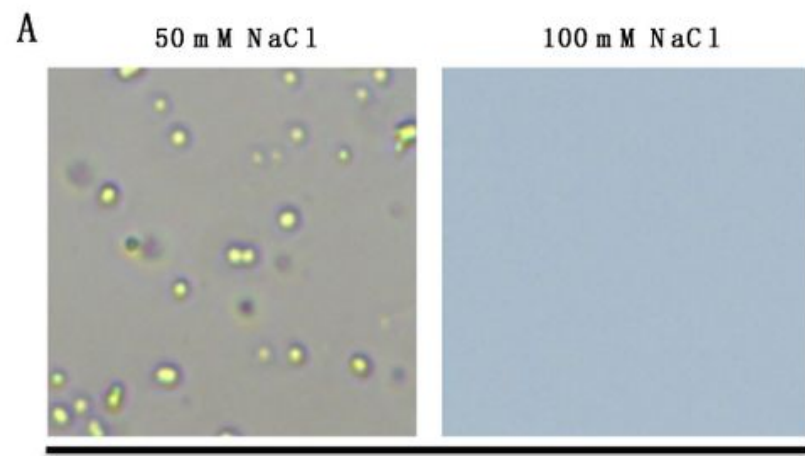

nsp8 din er $1 \mathrm{~m} \mathrm{~g} / \mathrm{m} 1$

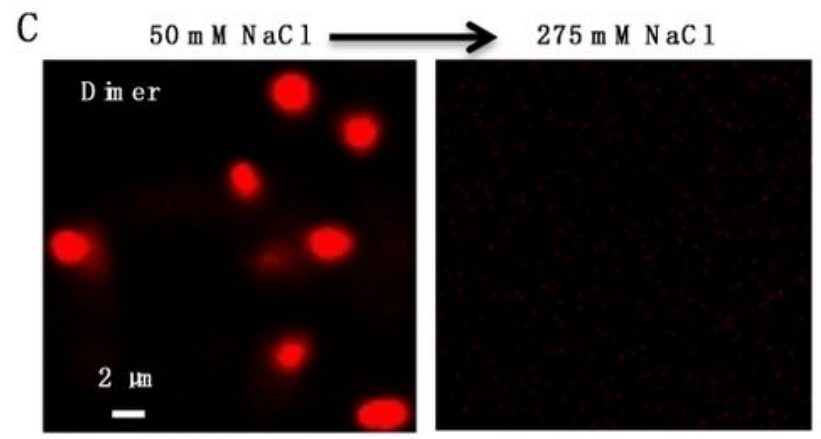

E

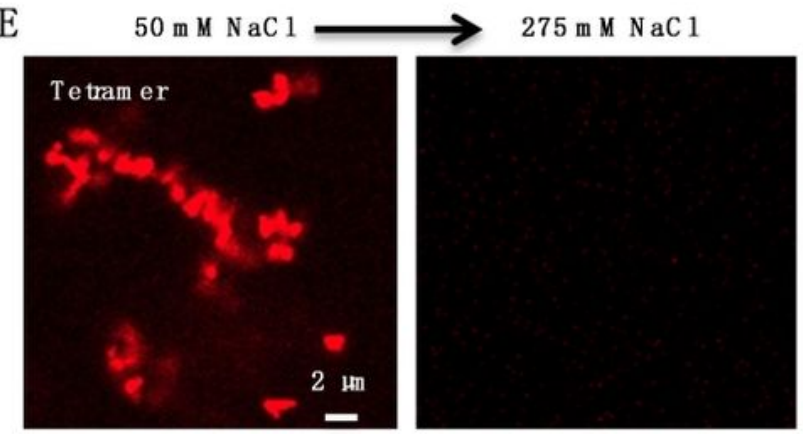

F

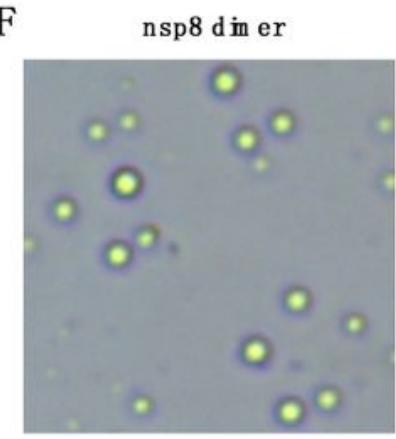

B

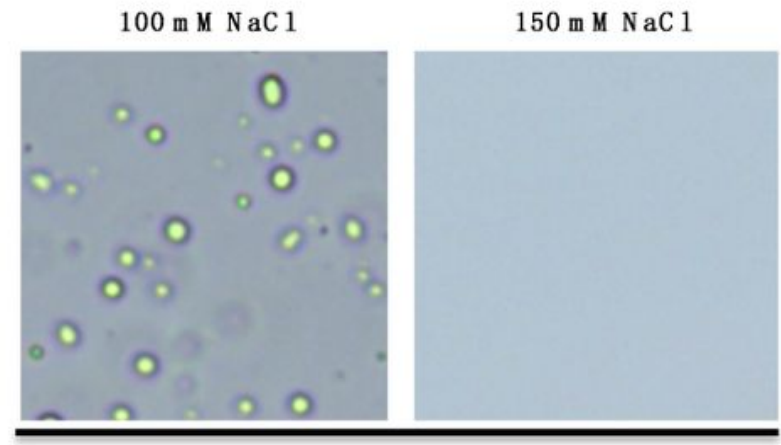

nsp8 din er2 m g/m 1

D
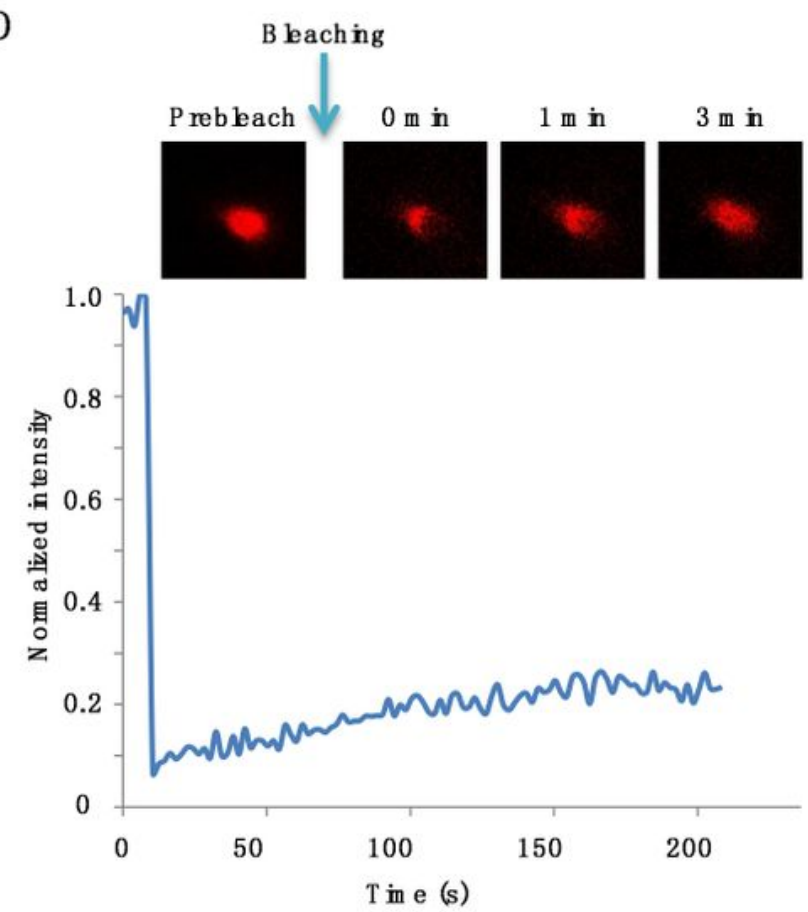

G

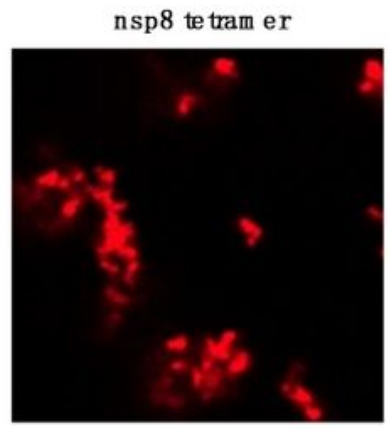

nsp8 $\Delta$ N 76 te tram er

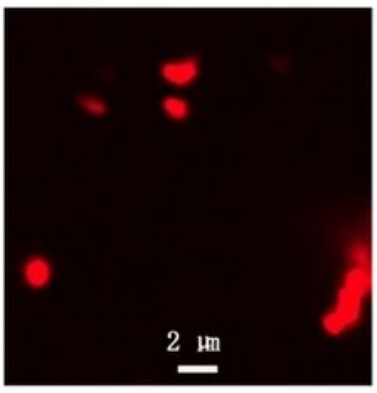

\section{Figure 2}

Phase separation of SARS-CoV-2 nsp8. (A) nsp8 form liquid-like droplets at $1 \mathrm{mg} / \mathrm{mL}$ in the buffer containing $50 \mathrm{mM} \mathrm{NaCl}$. (B) nsp8 form liquid-like droplets at $2 \mathrm{mg} / \mathrm{mL}$ in the buffer containing $100 \mathrm{mM}$ $\mathrm{NaCl}$. (C) Liquid-like droplets formed by RED-tris-NTA labeled nsp8 dimers can be dissolved upon 
increasing $\mathrm{NaCl}$ concentration to $275 \mathrm{mM}$. (D) In vitro FRAP analysis of the condensates formed by nsp8 at $2 \mathrm{mg} / \mathrm{mL}$ in buffer containing $100 \mathrm{mM} \mathrm{NaCl}$. (E) nsp8 tetramer at $0.25 \mathrm{mg} / \mathrm{mL}$ forms solid-like condensates in the buffer containing $50 \mathrm{mM} \mathrm{NaCl}$. The solid-like condensates also can be dissolved upon increasing $\mathrm{NaCl}$ concentration. $(\mathrm{F})$ Deletion of $\mathrm{N}$ terminal 76 residues inhibits the phase separation of nsp8 dimer. (G) Deletion of $\mathrm{N}$ terminal 76 residues reduces the aggregation of nsp8 tetramer.

A

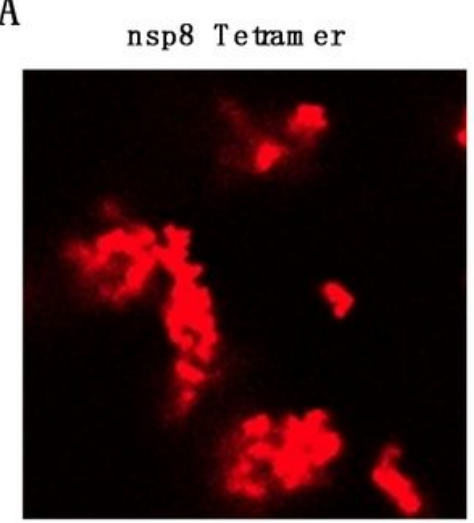

B

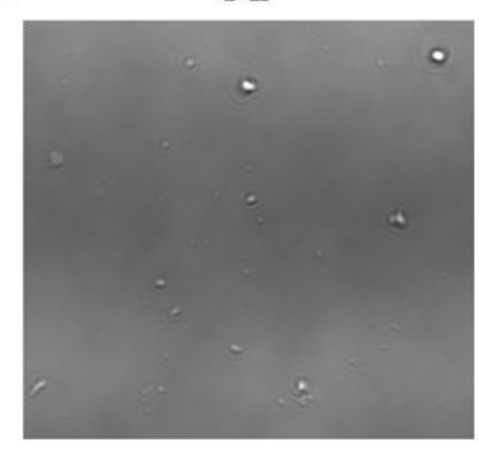

$+\mathrm{R} 12(75 \quad \mathrm{M})$

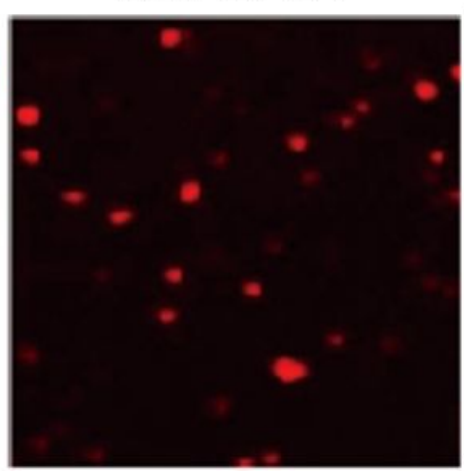

nsp8 Tetram er

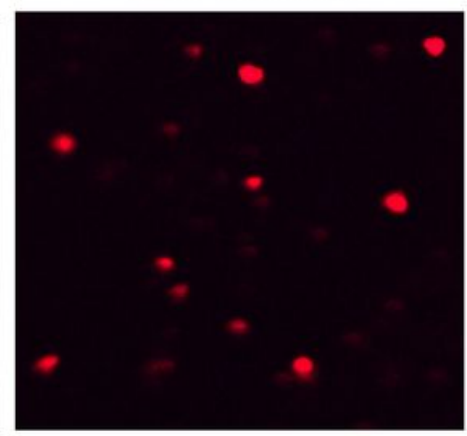

$+\mathrm{U} 12(75$ M $)$

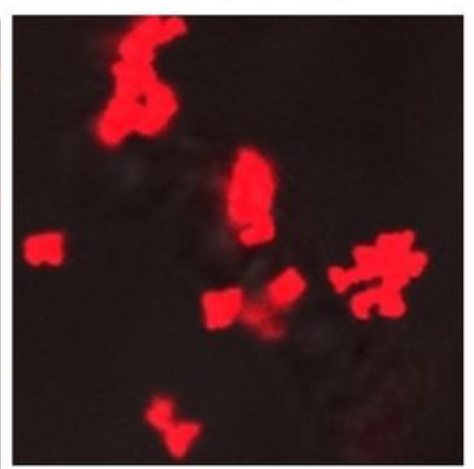

R 12

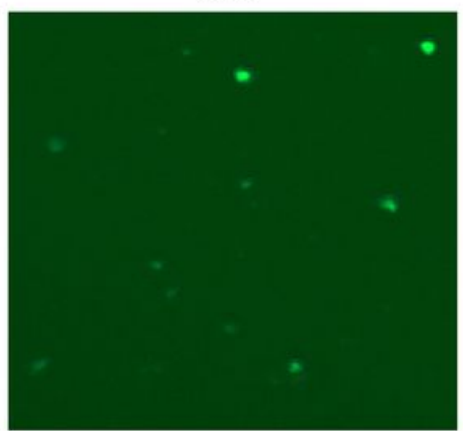

+ U22（7.5 M )

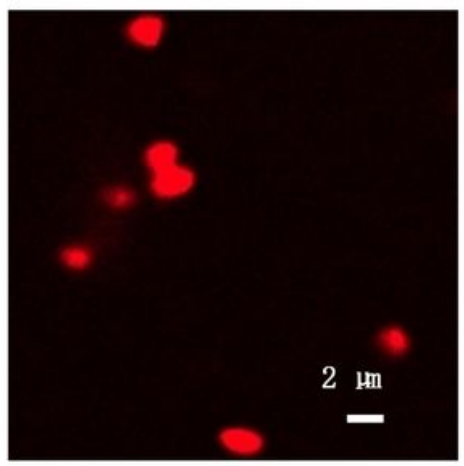

m erged

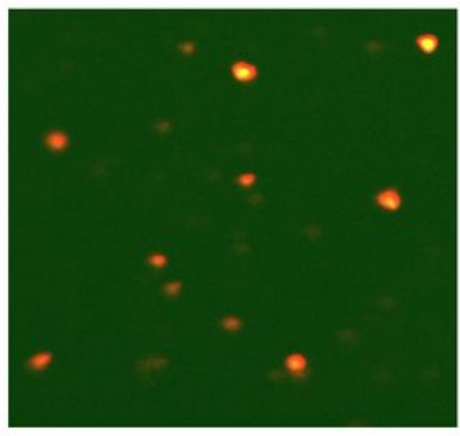

\section{Figure 3}

RNA modulates phase separation behaviors of nsp8 tetramer. (A) RNA induces LLPS of nsp8 tetramer in a sequence and length dependent manner. (B) Co-localization of nsp8 tetramer and ssRNA R12 in liquidlike droplets. Before imaging, $0.25 \mathrm{mg} / \mathrm{mL}$ RED-tris-NTA labeled nsp8 tetramer was mixed with $75 \mu \mathrm{M} 6$ FAM labeled R12 in buffer of $20 \mathrm{mM} \mathrm{HEPES} \mathrm{pH} \mathrm{7.4,50} \mathrm{mM} \mathrm{NaCl.}$ 


\section{Figure 4}

nsp8 forms condensates in live Hela cells.

\section{Supplementary Files}

This is a list of supplementary files associated with this preprint. Click to download.

- supplementarymaterial.docx 